\title{
ON THE TOPOLOGY OF RIEMANNIAN MANIFOLDS WHERE THE CONJUGATE POINTS HAVE A SIMILAR DISTRIBUTION AS IN SYMMETRIC SPACES OF RANK 1
}

\author{
BY WILHELM KLINGENBERG
}

Communicated by R. Bott, August 23, 1962

1. Manifolds similar to spheres.

1.1. Let $S=S^{n}$ be the $n$-dimensional sphere, endowed with the usual metric of constant Riemannian curvature 1. Let $G=(p(s))$, $0 \leqq s<\infty$, be a geodesic ray in $S^{n}, s$ being the arc length. Then the conjugate points of $p(0)$ on $G$ occur at $s=l \pi, l$ a positive integer, with multiplicity $n-1$.

Let $G$ be a geodesic ray in a Riemannian manifold $M=M^{n}$ of dimension $n$. The following condition may be interpreted, at least for $k=n-1$, as saying that the first $k$ conjugate points on $G$ are similarly distributed as on the sphere $S^{n}$ :

$(\Sigma, k)$ There are no conjugate points in the interval $[0, \pi[$ and at least $k$ conjugate points in $[\pi, 2 \pi[$, each counted by its multiplicity.

1.2. The following proposition gives a sufficient, but not necessary condition for the validity of $(\Sigma, n-1)$. For the proof see Morse [5].

Proposition 1. Let $G=(p(s))$ be a geodesic ray in a Riemannian manifold $M^{n}$. Assume that the Riemannian curvature $K(\sigma)$ of a plane section $\sigma$, tangent to $G$ at a point $p(s)$ with $s \leqq 2 \pi$ satisfies

$$
1 / 4<K(\sigma) \leqq 1
$$

Then $(\Sigma, n-1)$ holds for $G$.

1.3. We now study the implications of $(\Sigma, k)$ :

Lemma 1. Let $M=M^{n}$ be a simply connected, complete Riemannian manifold and assume that there is a point $o \in M$ such that for each geodesic ray, starting at $o,(\Sigma, k)$ holds with $k \geqq 2$. Then $M$ is compact. There is a point $o^{\prime} \in M$ with $d\left(o, o^{\prime}\right) \sim 0$ and not conjugate to $o$ such that each geodesic from $o$ to $o^{\prime}$ which is not the geodesic of minimal length $d\left(o, o^{\prime}\right)$ has length $\geqq 2 \pi-d\left(o, o^{\prime}\right) \sim 2 \pi$ and, therefore, has an index $\geqq k$.

Hence, the loop space $\Omega\left(o, o^{\prime}\right)$ has the homotopy type of a 0-cell to which there are attached cells of dimension $\geqq k$.

The proof of this lemma goes along the same lines as the proof of the lemma in [4] except that Rauch's comparison theorem is replaced by an application of the Gauss lemma. 
1.4. Using standard facts in Morse theory we have

THEOREM 1. If $M$ is an n-dimensional, simply connected, complete Riemannian manifold with the property that there is a point $o \in M$ such that each geodesic ray starting from o satisfies $(\Sigma, k)$, with $k \geqq 2$, then $\pi_{i}(M)=0$ for $1 \leqq i \leqq k$.

If here $(n-1) / 2 \leqq k$, then Poincare duality and standard facts in homotopy theory yield that $M$ has the homotopy type of the sphere and hence, according to Smale [7], $M$ is actually homeomorphic with the sphere, at least for $n \neq 3$ and $n \neq 4$.

\section{Combined with Proposition 1 we get}

TheOREM 2. Let $M^{n}$ be a complete, simply connected Riemannian manifold. If there is a point $o \in M$ such that for each plane section $\sigma$, tangent to one of the geodesic segments of length $2 \pi$ emanating from 0 , the Riemannian curvature $K(\sigma)$ satisfies (1), then $M^{n}$ is compact and has the homotopy type of the sphere and even is, at least for $n \neq 3,4$, homeomorphic with the sphere.

1.5. Presumably, under the assumptions of Theorem $2, M^{n}$ is homeomorphic to the sphere for all $n$. At least, this is the case when the assumptions do hold for all $o \in M$ or, what is the same, if (1) holds for all plane sections $\sigma$ of $M$. As this follows was shown in [4], from an argument provided by Berger [1]. A variation of this argument was given independently by Toponogov [9]. Both proofs are based on the information on the length of closed geodesics as provided by Lemma 1 and on Toponogov's theorem on geodesic triangles (cf. [8]). Tsukamoto [10] gave a third proof in which only an infinitesimal version of the triangle theorem is used which is due to Rauch [6].

2. Manifolds similar to one of the other compact, irreducible symmetric spaces of rank 1 .

2.1. Recall that these spaces belong to one of the following classes (cf. Cartan [2]):

The complex projective space, $P(1)^{n}$, having a dimension $n=2 m$ $\geqq 4$.

The quaternion projective space, $P(3)^{n}$, having a dimension $n=4 m \geqq 8$.

The projective Cayley plane, $P(7)^{n}$, having the dimension $n=16$.

These spaces shall be endowed with their usual metric in which the values of the Riemannian curvature vary between 1 and $1 / 4$. Let $G=$ $(p(s))$ be a geodesic ray in the space $P(\alpha)^{n}, \alpha \in\{1,3,7\}$. Then the 
conjugate points of $p(0)$ on $G$ occur at $s=(2 l-1) \pi, l$ a positive integer, with multiplicity $\alpha$, and at $s=2 l \pi, l$ a positive integer, with multiplicity $n-1$.

2.2. The following condition may be paraphrased by saying that the distribution of the conjugate points is, to the given degree, similar to the distribution which occurs in the symmetric space $P(\alpha)^{n}, \alpha \in\{1,3,7\}: \Pi(\alpha, n)$. There are no conjugate points in the intervals $[0, \pi[$ and $[5 \pi / 4,2 \pi[$, there are $\alpha$ conjugate points in $[\pi, 5 \pi / 4[$ and there are $n-1$ conjugate points in $[2 \pi, 5 \pi / 2[, n=(\alpha+1) m$, each counted with its multiplicity.

2.3. Let $o$ be a point in a complete Riemannian manifold $M=M^{n}$. Consider the exponential map exp: $M_{0} \rightarrow M$ of the tangent space $M_{0}$ of $M$ at $o$ onto $M$. To each ray $\bar{G}=(\bar{p}(s)), 0 \leqq s<\infty$, in $M_{0}$, starting from the origin $o \in M_{0}$, there corresponds the geodesic ray $G=(p(s))$, $0 \leqq s<\infty$, in $M$, starting from $o \in M$ in the same direction as $G$. Then $\bar{p}(s)$ is a critical point for the exponential map if and only if $p(s)$ is a conjugate point on $G$.

We use this well-known fact to explain what it means that $\Pi(\alpha, n)$ holds for all geodesic rays starting from $o$; later we will see that this property has far reaching consequences for the topology of $M$.

Denote by $B(s)$ the open ball in $M_{0}$ of radius $s$ and center at the origin $o \in M_{0}$. Then our assumption implies, first of all, that there are no critical points for exp in $B(\pi)$. In contrast, a ray $\bar{G}$ passing through $D=B(5 \pi / 4)-B(\pi)$, will hit $\alpha$ critical points; we like to think, therefore, of $D$ as of some sort of van Allen radiation belt. But once we are beyond this belt we reach again a safe region $E=B(2 \pi)-B(5 \pi / 4)$ without critical points. The far side of $E$, however, is again surrounded by a dangerous belt, i.e., $B(5 \pi / 2)-B(2 \pi)$, which is thickly populated ( $n-1$ on each ray!) with critical points.

It is the safe belt $E$, beyond the first dangerous belt $D$, which constitutes the essential new feature compared with the situation considered in Chapter 1.

Note that for the symmetric space $P(\alpha)^{n}$ the two dangerous belts are squeezed together into spheres of radius $\pi$ and $2 \pi$, respectively.

The condition $\Pi(\alpha, n)$ may be interpreted as a certain perturbation of this highly degenerate and unstable situation. Of course, one may consider even stronger perturbations than the one described by $\Pi(\alpha, n)$. However, the results we are able to draw in such a case are less conclusive than the one presented below.

2.4. The following proposition gives a sufficient but not necessary condition for the validity of $\Pi(1, n)$ in a Kähler manifold $M=M^{n}$. First we recall that a plane section $\sigma$ in $M$ determines an angle 
$\omega=\omega(\sigma), 0 \leqq \omega \leqq \pi / 2$, in the following way: If $X$ is a vector $\neq 0$ in $\sigma$, let $\bar{\sigma}$ be the 2-plane spanned by $X$ and $J X, J$ being the imaginary operator; then $\omega(\sigma)$ is defined as the angle between $\sigma$ and $\bar{\sigma}$.

For the complex projective space $P(1)$ the Riemannian curvature $K(\sigma)$ of a plane section $\sigma$ depends only on the angle $\omega=\omega(\sigma)$ and is given by $K_{1}(\omega)=\left(1+3 \cos ^{2} \omega\right) / 4$.

Proposition 2. Let $M^{n}$ be a Kähler manifold. Let $G=(p(s))$ be a geodesic ray in $M^{n}$. Assume that the Riemannian curvature $K(\sigma)$ of a plane section $\sigma$, tangent to $G$ at a point $s \leqq 2 \pi$ satisfies

$$
0.64 K_{1}(\omega(\sigma))<K(\sigma) \leqq K_{1}(\omega(\sigma)) .
$$

Then $\Pi(1, n)$ holds for $G$.

The proof follows from the index theorem of Morse [5].

2.5. We now give the implications of $\Pi(\alpha, n), \alpha \in\{1,3,7\}$.

LEMMA 2. Let $M^{n}$ be a simply connected, complete Riemannian manifold and assume that there is a point $o \in M$ such that $\Pi(\alpha, n)$ holds for each geodesic ray starting from 0 . For $\alpha=1$ assume, in addition, that there is a point in $M$ which has distance $\pi$ from 0 . We note that this is the case if $M$ has positive Riemannian curvature for all plane sections (cf. [3]). Then the following does hold:

(i) $M$ is compact.

(ii) There is a point $o^{\prime} \in M$ with $d\left(o, o^{\prime}\right) \sim 0$ and not conjugate to $o$ such that the loop space $\Omega\left(o, o^{\prime}\right)$ contains no geodesic of index $i$ with $0<i<\alpha$.

(iii) There is a point $o^{\prime \prime} \in M$ with $d\left(o, o^{\prime \prime}\right) \sim \pi$ and not conjugate to o such that the loop space $\Omega\left(o, o^{\prime \prime}\right)$ contains only geodesics which either have length $<5 \pi / 2-d\left(o, o^{\prime \prime}\right) \sim 3 \pi / 2$ and hence have an index $\leqq \alpha$ or have length $>3 \pi / 2+d\left(o, o^{\prime \prime}\right) \sim 5 \pi / 2$ and hence have an index $\geqq n-1$ $+\alpha$. Furthermore, the subspace $\Omega^{2 \pi}$ of $\Omega\left(o, o^{\prime}\right)$, formed by the curves of length $\leqq 2 \pi$ (which contains only geodesics of index $\leqq \alpha$ ) has the homotopy type of the $\alpha$-sphere.

(iv) The loop space of $M$ has the homotopy type of an $\alpha$-sphere to which there are attached cells of dimension $>n-2+\alpha$.

(i) is clear. (ii) follows from Lemma 1 which, for $\alpha=3,7$, also yields the existence of a point with distance $\pi$ from $o$.

To prove (iii) we introduce the subspace $\tilde{\Omega}=\tilde{\Omega}\left(o, o^{\prime \prime}\right)$ of $\Omega=\Omega\left(o, o^{\prime \prime}\right)$ consisting of those curves which start out from $o$ as a geodesic segment of length $5 \pi / 4$ and then continue to $o^{\prime \prime}$. On $\tilde{\Omega}$ we consider the length function. Then there are two types of critical points: First, the geodesic segments from $o$ to $o^{\prime \prime}$ of length $\geqq 5 \pi / 4$; their index in 
$\tilde{\Omega}$ is $\alpha$ units less than it is in $\Omega$. Second, there are the once broken geodesic segments of the following form: They start as a geodesic from $o$ to $o^{\prime \prime}$ of length $<5 \pi / 4$ and then go on beyond $o^{\prime \prime}$ until they reach the length $5 \pi / 4$ and then they return the same way back to $o^{\prime \prime}$. The index in $\tilde{\Omega}$ of such a critical point is given by the number of conjugate points on the initial segment of length $5 \pi / 4$ which occur after $o^{\prime \prime}$. That means: If the initial segment from $o$ to $o^{\prime \prime}$ of length $<5 \pi / 4$ has index $i$ in $\Omega$, the broken segment has index $\alpha-i$ in $\tilde{\Omega}$.

The statements in (iii) on the length of geodesics in $\Omega\left(o, o^{\prime \prime}\right)$ are now proved with the help of a lifting argument for a homotopy between two critical points in $\tilde{\Omega}$, similar to the one used in the proof of Lemma 1. This time, however, the lifting of the curves of $\tilde{\Omega}$ into $M_{0}$ does not give curves in $B(\pi)$ but gives curves which start out from $o \in M_{0}$ as a straight segment of length $5 \pi / 4$ which brings them into the safe region $E=B(2 \pi)-B(5 \pi / 4)$ (cf. 2.3) where they then continue to stay until either they fall back into the van Allen belt $D$ (cf. 2.3) which is the uninteresting case or until they reach the outer border of $E$ at a distance $2 \pi$.

The last statement in (iii) is proved by noting that $\Omega^{2 \pi}$ and $\tilde{\Omega}^{2 \pi}$ have the same homotopy type but yield CW-complexes which are dual to each other. Standard facts then give (iv).

2.6. Property (iv) in Lemma 2 implies that $M^{n}$ and $P(\alpha)^{n}$ have the same homotopy groups up to dimension $n-1+\alpha$. A spectral sequence argument gives that $M^{n}$ and $P(\alpha)^{n}$ have the same integer cohomology ring. Combining this with Proposition 2 we get

THEOREM 3. Let $M^{n}, n \geqq 4$, be a complete Kähler manifold and assume that the Riemannian curvature $K(\sigma)$ of the plane sections $\sigma$ of $M^{n}$ satisfies (2). Then $M^{n}$ has the homotopy type of the complex projective space $P(1)^{n}$.

Indeed, since $\pi_{i}\left(P(1)^{n}\right)=0$ for $3 \leqq i \leqq n$, it is possible to extend a map of the 2-skeleton of $M$ into $P(1)$ to a map of $M$ into $P(1)$. In particular, this can be done such as to imply an isomorphism of the cohomology ring. But then $M$ and $P(1)$ have the same homotopy type (cf. Whitehead [11]).

On the other hand, for $\alpha=3,7$, we have at least

THEOREM 4. Let $M$ be a simply connected, complete Riemannian manifold of the same dimension as the symmetric space $P(\alpha)^{n}, \alpha \in\{3,7\}$. If there is a point $o \in M$ such that $\Pi(\alpha, n)$ holds for each geodesic ray starting at o then $M$ is compact and has the same integer cohomology ring as $P(\alpha)^{n}$. 


\section{REFERENCES}

1. M. Berger, Les variêtés riemanniennes (1/4)-pincées, C. R. Acad. Sci. Paris 250 (1960), 442-444.

2. E. Cartan, Sur certaines formes riemanniennes remarquables des géométries d groupe fondamental simple, Ann. Sci. Ecole Norm. Sup. 44 (1927), 345-467.

3. W. Klingenberg, Contributions to Riemannian geometry in the large, Ann. of Math. (2) 69 (1959), 654-666.

4. - Über Riemannsche Mannigfaltigkeiten mit positiver Krümmung, Comment. Math. Helv. 35 (1961), 47-54.

5. M. Morse, The calculus of variations in the large, Amer. Math. Soc. Colloq. Publ. Vol. 18, Amer. Math. Soc., Providence, R. I., 1934.

6. H. E. Rauch, $A$ contribution to differential geometry in the large, Ann. of Math. (2) 54 (1951), 38-55.

7. S. Smale, The generalized Poincaré conjecture in higher dimensions, Bull. Amer. Math. Soc. 66 (1960), 373-375.

8. V. A. Toponogov, Riemannian spaces which have their curvature bounded from below by a positive number, Dokl. Akad. Nauk SSSR 120 (1958), 719-721. (Russian)

9. - Dependence between curvature and topological structure of Riemann spaces of even dimension, Dokl. Akad. Nauk SSSR 133 (1960), 1031-1033. (Russian)

10. Y. Tsukamoto, On Riemannian manifolds with positive curvature, Mem. Fac. Sci. Kyushu Univ. 15 (1962), 90-96.

11. J. H. C. Whitehead, Combinatorial homotopy. I, Bull. Amer. Math. Soc. 55 (1949), 213-245.

University of CALIFornia, Berkeley 